\title{
Estrategias metacognitivas en la comprensión auditiva del inglés, caso colegio municipal Fernández Madrid
}

\author{
Metacognitive strategies in the auditory comprehension of English, municipal school \\ case Fernández Madrid
}

\section{Estratégias metacognitivas na compreensão auditiva do inglês, caso da escola municipal Fernández Madrid}

Myriam M. Hurtado-Angamarca ${ }^{\mathrm{I}}$

moryhurt@hotmail.com

Amilcar O. González-Díaz II

aogonzalez@uce.edu.ec

Gabriela M. Moso-Mena ${ }^{\text {III }}$

gmmoso@uce.edu.ec

Edison S. Sanguña-Loachamin IV

essanguna@uce.edu.ec

Recibido: 30 de enero de $2017 *$ Corregido: 20 de febrero de $2017 *$ Aceptado: 20 junio de 2017

I. Magister en Lingüística y Didáctica de la Enseñanza de Idiomas Extranjeros; Licenciada en Ciencias de la Educación Mención Ingles; Universidad Central del Ecuador, Quito, Ecuador.

II. Magister en Lingüística y Didáctica de la Enseñanza de Idiomas Extranjeros; Diploma Superior de la Enseñanza de Inglés como Segunda Lengua; Licenciado en Ciencias de la Educación Mención Ingles; Universidad Central del Ecuador, Quito, Ecuador.

III. Magister en Educación Superior y Equidad de Género; Licenciada en Ciencias de la Educación Mención Plurilingüe; Universidad Central del Ecuador, Quito, Ecuador.

IV. Magister en Docencia Universitaria y Administración Educativa; Especialista en Diseño Curricular por Competencias; Licenciado en Ciencias de la Educación Mención: Plurilingüe; Universidad Central del Ecuador, Quito, Ecuador. 


\section{Resumen}

El presente trabajo investigativo tiene como propósito principal comprobar si existe la aplicación de estrategias metacognitivas para el desarrollo de la comprensión auditiva del inglés. Como primer aspecto relevante se evidencia la literatura científica sobre el tema, posterior a esto se elaboró tres instrumentos para la recolección de datos. Se realizó la encuesta a los docentes del área de inglés con el propósito de medir su nivel de conocimiento sobre metacognición y las estratégias metacognitivas. De igual manera, se construyó una prueba de comprensión auditiva y se aplicó el cuestionario que mide la consciencia metacognitiva sobre la habilidad de escuchar en inglés (MALQ). El análisis e interpretación de los datos proyectó que los docentes tienen un buen nivel de conocimiento sobre metacognición, sin embargo no aplican metacognición durante sus clases en una actividad auditiva. Esto es comprobable con los datos que demuestran tanto la prueba de comprensión auditiva como los resultados del cuestionario metacognitivo que señalan que una gran mayoría de estudiantes no conoce sobre estrategias metacognitivas para mejorar la comprensión auditiva del inglés. En consecuencia se plantea una guía estratégica para promover el desarrollo de dichas estrategias en los estudiantes y su aplicación por parte de los docentes.

Palabras clave: Metacognición; comprensión auditiva; estrategia; actividad metacognitive; MALQ. 


\section{Abstract}

The main objective of the current research work is to investigate whether students apply metacognitive strategies to develop the listening comprehension skill of English. The scientific literature about the topic represents the first relevant aspect in this work. The second important element has to do with three evaluation tools construction developed to collect the data and information about it. In order to identify the levels of metacognitive knowledge of the English teachers, a survey was conducted. To see if students use metacognitive strategies and analyze their listening comprehension performance of English a listening activity test and the metacognitive awareness listening questionnaire (MALQ) were conducted. The analysis and interpretation of the data demonstrated that the teachers' knowledge about metacognition is widely acceptable and high; however there is no application of these metacognitive strategies during a listening activity process during classes. This fact is greatly tested by means of the listening activity test and the results obtained from the metacognitive awareness listening questionnaire whose results attribute that the majority of students do not use metacognitive strategies to improve listening comprehension. Consequently a strategic guide is proposed to hone the development of metacognitive strategies for students and its application by means of teachers.

Key words: Metacognition; listening comprehension; strategy, metacognitive activity; MALQ. 


\section{Introducción.}

Es innegable que en este mundo globalizado en el cual vivimos, aprender inglés es una de las llaves de entrada a una infinita cantidad y variedad de información y conocimientos. Según Cogo \& Dewey (2012), el inglés se ha masificado y es usado por una gran diversidad de ciudadanos de distintos países que no tienen este idioma como lengua materna. La expansión del idioma inglés es a escala global y es usado en todo tipo de intercambio de información.

A pesar de la importancia del idioma inglés, la problemática del aprendizaje de este idioma está latente en las instituciones del país. Con la presente investigación se pretende dar tratamiento y solucionar el problema del desarrollo de la comprensión auditiva del inglés, ya que el bajo nivel de comprensión de este idioma, en nuestros estudiantes, se debe a la falta de estrategias enfocadas a mejorar la destreza de escuchar. De acuerdo con Modi (1991) “ la destreza de escuchar es importante no solo porque es la manera como se aprende un idioma, sino porque es el proceso básico a través del cual se aprende pronunciación, vocabulario y se obtiene una idea de la organización del idioma, es decir la sintaxis" (p.2).

Con este trabajo de investigación se espera obtener resultados positivos sobre el desarrollo de la destreza de escuchar en inglés, pues a través del uso de estrategias metacognitivas por parte de los estudiantes se puede lograr su participación activa, ya que el eje fundamental de este enfoque se basa en la reflexión, y la autoconciencia sobre su propio aprendizaje. Las estrategias metacognitivas de acuerdo con Huseyin (2005) juegan un papel fundamental en el aprendizaje exitoso, pues guían los procesos de pensamiento de cada persona y pueden ser utilizadas para ayudar a los individuos a entender cómo aprenden y cómo adquieren el aprendizaje. 
Nuestro país se encuentra en un proceso de cambios a nivel social, económico, tecnológico, educativo, entre otros. En el aspecto educativo, estos cambios se evidencian en el nuevo currículo del Idioma Inglés alineado al Marco Común Europeo, este currículo está desarrollado para formar individuos capaces de comunicarse en inglés que puedan acceder a la sociedad del conocimiento y enfrentar los retos que se deriven de esta. Núñez Perez, Solano Pizarro, González-Pienda, \& Rosário (2006) en su artículo educativo mencionan que lo más importante en este ámbito, es que los individuos estén preparados para ser autónomos en su propio aprendizaje, es decir, desarrollar competencias que les permitan formarse por sí solos y a lo largo de sus vidas. En este marco de importancia este trabajo investigativo se relaciona con su aspecto socioeducativo, ya que el objetivo para los estudiantes es mejorar el aprendizaje del idioma inglés para su exitosa inserción en la sociedad.

En este sentido es cada vez más relevante que nuestros educandos mejoren sus capacidades de comprender lo que escuchan y tengan plena conciencia de este proceso cognitivo, para que su enriquecimiento intelectual sea infinito. Esto se logrará incorporando a sus labores diarias estrategias metacognitivas que los ayuden a "aprender a aprender" y "aprender a pensar".

La presente investigación tiene como objetivo determinar la efectividad de las estrategias metacognitivas en la comprensión auditiva del inglés en los estudiantes del segundo año de bachillerato del Colegio Municipal Fernández Madrid. 


\section{Materiales y métodos.}

\section{Diseño de la investigación}

Determinar de qué manera las estrategias metacognitivas dentro de los procesos de enseñanza inciden en la compresión auditiva del idioma inglés es la finalidad de esta investigación, en este sentido ya se planteó el marco teórico que justifica, enmarca y contextualiza los porqués de este trabajo investigativo.

Está claro que este tipo de estrategias educativas son en esencia activas pues requieren una participación activa del estudiante dentro de los procesos de aula (Tarricone, 2011). Este fenómeno es el que se midió, tabuló y sobre el cual se concluyó tomando en cuenta los siguientes aspectos metodológicos en la investigación:

La investigación tuvo una modalidad cualitativa, pues la descripción del fenómeno se basó en hechos que no dieron paso a interpretaciones subjetivas de los sujetos, sino al contrario las preguntas formuladas y las respuestas obtenidas fueron perfectamente medibles en un ambiente controlado, como es el aula como espacio de trabajo.

Otro de los aspectos de la investigación es que esta no fue experimental sino tan solo descriptiva, es decir, el investigador observó una o varias circunstancias ocurridas, examinó las relaciones entre los aspectos sin ninguna manipulación directa de las condiciones que son experimentales (Jaramillo \& Ramírez, 2006). Esto implicó; una conducta normal de los sujetos dentro de un ambiente cotidiano de trabajo, por tanto, la descripción tan solo refirió a los múltiples comportamientos del fenómeno existente utilizando números para caracterizar tanto a los individuos como al grupo y evalúo la naturaleza de las condiciones existentes. Por último el propósito de este 
trabajo fue caracterizar algo como es y evidenciar al detalle las múltiples aristas de este problema para arribar a todas las posibles soluciones.

Mediante la aplicación de instrumentos y técnicas lo que se pretende es mostrar, lo que empíricamente es evidente, que no se usan estrategias metacognitivas dentro del aula en los procesos de comprensión auditiva del idioma inglés.

El imperativo del presente trabajo es determinar dentro de la comunidad educativa, como una necesidad ineludible, las estrategias metacognitivas en la comprensión auditiva del inglés y cómo estas contribuyen al desarrollo de la adquisición de una lengua extranjera.

\section{Enfoque de la investigación}

El enfoque de la investigación se relaciona con los criterios cualitativo y cuantitativo, y hacen referencia a dos modalidades de estudio o de aproximación al fenómeno, al objeto de estudio, así mismo caracterizan dos niveles de discurso sobre la naturaleza de abordaje del conocimiento, de cómo el investigador entiende el mundo y el objetivo último del estudio a realizarse.

Estos enfoques en cuanto al fenómeno investigado por lo general ejecutan las siguientes fases similares y relacionadas entre sí: observación y evaluación de fenómenos; determinar suposiciones o ideas como consecuencia de la observación y evaluación realizada; probar y demostrar el grado en que las suposiciones o ideas tienen fundamento; y proponer nuevas observaciones y evaluaciones para esclarecer, modificar, cimentar y/o fundamentar las suposiciones e ideas; o incluso para generar otras. 
La diferencia básica de estos dos enfoques es la presentación de los datos; el enfoque cualitativo presenta los datos en forma narrativa y de cierta manera subjetiva; mientras que el enfoque cuantitativo presenta los resultados estadísticos en forma de números.

En el enfoque cuantitativo, utilizado en la presente investigación, se recolectó y analizó datos para contestar preguntas de investigación; ¿Cuál es el aporte de las estrategias metacognitivas para el desarrollo de la comprensión auditiva del inglés?, ¿Qué tipo de estrategias metacognitivas mejorarán la comprensión auditiva del inglés?, ¿En qué medida la guía didáctica de talleres sobre estrategias metacognitivas ayudará a mejorar la comprensión auditiva del inglés? y probar el supuesto de que la comprensión auditiva mejorará con el uso de estrategias metacognitivas.

Además, como ya se mencionó, en la investigación se abordó el fenómeno de estudio de forma no experimental y descriptiva, esto relacionado con el problema que planteado: ¿De qué manera las estrategias metacognitivas inciden en la comprensión auditiva del inglés... significa que se describió el comportamiento -nuevo- frente a la frecuencia de uso de las estrategias metacognitivas en la enseñanza del inglés, y se examinó además las relaciones entre los aspectos sin ninguna manipulación directa de las condiciones normales de trabajo de los estudiantes, contrastando los resultados entre el uso de estrategias metacognitivas y el trabajo rutinario sin el uso de estas.

Este tipo de investigación refiere simplemente a un fenómeno existente utilizando números para caracterizar individuos y grupos, esto parte de la premisa de que hay hechos sociales relativamente estables y por tanto medibles: el proceso de comprensión auditiva en general y el uso de estrategias metacognitivas en la enseñanza del inglés en específico, estos fueron abordados lejos de los sentimientos y las opiniones de las personas que tienen un alto grado de subjetividad y que 
corresponden más bien a una investigación cualitativa, por tanto, se caracterizó el fenómeno del uso de estrategias metacognitivas dentro del proceso auditivo del inglés.

Mediante la aplicación de instrumentos y técnicas se mostró, lo que empíricamente podría resultar evidente, que no se usa estrategias metacognitivas dentro del aula en la enseñanza de una lengua extranjera y en específico en el proceso auditivo del inglés lo que determina el nivel de aprendizaje y apropiación de la misma.

El imperativo del presente trabajo es determinar dentro de la comunidad educativa, como una necesidad ineludible el uso de estrategias metacognitivas en la comprensión auditiva del inglés.

\section{Modalidad de la Investigación}

La investigación, en cualquier campo o disciplina, tiene relación con diferentes formas de razonamiento; cómo se entiende y comprende una realidad, la significación que le otorga el sujeto a lo que estudia e investiga, la dirección y el sentido que le adjudica a su objeto de estudio, el grado de intervención del investigador.

Es importante precisar que a cualquier razonamiento o modalidad de investigación le sustenta un marco teórico desde el cual es posible tal razonamiento; es decir, el razonamiento se configura -intencionalmente o no- a partir de elementos de orden teórico-conceptuales, de perspectivas analíticas-interpretativas.

La investigación tuvo distintas etapas, en un primer momento fue bibliográfica documental, la cual abordó un sinfín de conceptualizaciones, clasificaciones, tesis, argumentos, 
teorías, posturas, autores acerca de la metacognición, sus antecedentes y las proyecciones teóricas y didácticas de este cuerpo teórico.

En un segundo momento se realizó la investigación de campo que de acuerdo con (Bayardo, 1987) esta forma de investigación necesariamente debe recurrir al contacto directo con los fenómenos de estudio, sea que los hechos estén suscitándose por la intervención del investigador a través del control de las variables o bien sin la intervención del investigador o de manera ajena a él. También se aplicó un enfoque descriptivo, cuantitativo y no experimental, para luego pasar a tabular e interpretar los datos obtenidos de las observaciones y finalmente se redactaron las conclusiones a las que derribamos luego de todo este proceso.

\section{Tipo de investigación}

Primero el objetivo de la presente investigación es la descripción objetiva de un fenómeno; la comprensión auditiva del inglés, lo cual responde a planteamientos como quién, qué, dónde, cuándo y cómo, y siendo una descripción objetiva se basó fundamentalmente en datos estadísticos.

La profundidad de la descripción de un fenómeno investigativo que se sustenta en el siguiente concepto: una vez que conocido el problema, hecho o situación, lo describe utilizando herramientas como la observación y estudios correlaciónales. Torres (2006) sobre los estudios correlacionales indica que el objetivo de esta es mostrar o examinar la relación que hay entre variables sin que necesariamente la una variable sea la causa de la otra. Este nivel de investigación acopia datos sobre hechos o fenómenos tal como se originan en la realidad con el objetivo de lograr interpretar y examinar las relaciones entre ellos. Dicho de otro modo la presente investigación pretende deducir si el uso de estrategias metacognitivas mejora la comprensión auditiva del inglés. 
Esta investigación también se sustenta en las preguntas directrices y los supuestos de investigación al cual se desean arribar.

Finalmente como ya se mencionó esta investigación tendrá un enfoque cuantitativo en cuanto a la presentación de la evidencia recogida en el proceso de investigación, y por último no experimental, pues las condiciones en que investigó el problema no fue manipulado.

\section{Población y muestra de investigación}

\section{Población}

De acuerdo con Tamayo (2004) la población se la define como: " La totalidad de un fenómeno de estudio, incluye la totalidad de unidades de análisis o entidades de población que integran dicho fenómeno" (p. 176). La población objetivo de la presente investigación es el universo de las y los estudiantes de segundo de bachillerato del Colegio Municipal Fernández Madrid del Distrito Metropolitano de Quito que integran un número de 192 educandos con quienes se trabajó para obtener un estudio mucho más representativo. En cuanto a las características de los individuos de estudio se puede mencionar que comparten particularidades como edad, intereses y rasgos sociales. Cabe indicar que se trabajó con el universo de estudiantes de segundo año de bachillerato para poder ampliar el campo de estudio y obtener datos mucho más relevantes.

En el caso de los maestros, tomando en cuenta el tema de la investigación alrededor de la asignatura de inglés se tomó como universo al conjunto de los profesores que imparten esta materia, que son un total de 7, y siendo una población menor a 30 miembros, se trabajó con todos. 


\section{Muestra de investigación}

De acuerdo con Hurtado y Toro (2007) "la muestra es un conjunto de elementos representativos de una población con quienes se trabajará realmente en el proceso de la investigación" (p.92). También nos informa que cuando no es posible medir cada una de las unidades de estudio de la población se aplican diversos métodos para obtener una muestra. Se toma una muestra de estudio cuando no se dispone de tiempo o recursos para estudiar a todo el universo y para un mejor manejo de los datos. De esta manera se pone de manifiesto que al tener la posibilidad de trabajar con toda la población de segundo año de bachillerato y poder aplicar sin complicaciones los instrumentos de investigación no hubo necesidad de determinar una muestra de estudio. Por otro lado la confiabilidad y la concordancia con las especificaciones que se deseó observar se consideraron para trabajar con todo el universo de estudiantes.

\section{Técnicas e instrumentos de recolección de datos}

En esta dimensión de la metodología se resuelve cuestiones vinculadas al proceso de obtención de información así como fuentes apropiadas que permitan obtener datos que luego se convertirán en significativos para el presente trabajo.

En primer lugar se destaca el trabajo realizado con los estudiantes a quienes se aplicó dos instrumentos para recolección de datos. En la literatura científica se menciona sobre lo que comprende el Marco Común Europeo de Referencia de las Lenguas Extranjeras, el mismo que en nuestro país está siendo utilizando para desarrollar el currículo para la enseñanza del inglés. Por lo tanto una prueba de comprensión auditiva enmarcada en este trabajo se elaboró de acuerdo al nivel de los estudiantes y a los conocimientos que hasta ahora poseen. La prueba requería que los 
educandos escuchen seis conversaciones o narraciones para verificar su comprensión a través de 30 preguntas de opción múltiple, identificación y completación que tenían el valor de un punto cada una. El objetivo de la aplicación de este instrumento de recolección de datos era medir la proficiencia de los estudiantes en cuanto a su comprensión auditiva.

Por otro lado, de acuerdo con Torres (2006) el cuestionario es de vital importancia en la investigación científica, ya que es el recurso más utilizado y a veces el único para obtener información. Permite estandarizar y uniformar la recopilación de datos. Una elaboración apropiada del cuestionario permite obtener datos precisos y confiables. De estas características partimos para la utilización de un cuestionario que está aprobado y validado internacionalmente desde que el investigador Vandergrift en el año 2006 diseñara el cuestionario Metacognitive Awareness Listening Questionnaire (MALQ), que en español significa Cuestionario Auditivo de Consciencia Metacognitiva. Este instrumento está basado en la teoría de Flavell sobre metacognición y en el modelo de Wenden sobre aprendizaje de segundas lenguas (1998). Los cuales proveen conocimiento sobre validación teórica en la construcción de preguntas. Su confiabilidad y validez fueron examinados y determinados por un criterio estandarizado para cuestionarios desarrollado por (Brown, 2001; Dornyei, 2003; Gilham, “ 2000), este trabajo involucra examinar literatura científica sobre estrategias de aprendizaje, metacognición, comprensión auditiva y autorregulación (Dörnyei \& Taguchi, 2010). El cuestionario ha sido probado con varios estudios sobre percepciones de los mismos estudiantes al realizar una actividad de comprensión auditiva, su comprensión sobre las tareas y la conciencia sobre las estrategias que les facilitan la comprensión auditiva (Li, 2013).

El MALQ sus siglas en inglés, está compuesto por cinco factores o tipos de estrategias que están relacionados con los tres tipos de conocimiento metacognitivo que están plasmados en 21 
proposiciones que le permiten al estudiante reflexionar y registrar sus procesos mentales sobre lo que realizó en cada etapa de la prueba auditiva del inglés (Peña-Ayala, 2014).

Este cuestionario retrospectivo que significa permitirle al estudiante recordar la manera como llevó a cabo sus procesos cognitivos antes, durante y después de una actividad auditiva dentro del aprendizaje de una lengua extranjera, fue aplicado inmediatamente después de la prueba de comprensión auditiva mencionada anteriormente con el objetivo de determinar la efectividad del uso de estrategias metacognitivas en la comprensión auditiva del inglés y el tipo de estrategias que utilizan los estudiantes que si obtuvieron un buen nivel de comprensión auditiva en la prueba aplicada. El formato de este cuestionario dispone de cinco rangos en la escala de Likert desde totalmente de acuerdo hasta en desacuerdo.

Finalmente, se seleccionó la técnica de la encuesta para trabajar con los docentes del Colegio Fernández Madrid por lo que también se aplicó como instrumento de recolección de datos el cuestionario para explorar su nivel de conocimiento sobre metacognición y estrategias metacognitivas que poseen. Este trabajo se lo realizó con el objetivo de estructurar la guía didáctica de talleres sobre metacognición que está dirigida a profesores y estudiantes. En el diseño de este cuestionario constan veinte preguntas enfocadas a determinar los constructos relevantes del marco teórico los mismos que se ubicaron indistintamente para evitar la secuencia de temas. El cuestionario dispone de cinco criterios para la escala valorativa en donde 5 indica por completo de acuerdo y 1 significa por completo en desacuerdo. Este cuestionario mide la percepción de los docentes sobre el tema, mientras que el cuestionario MALQ evalúa el nivel de conciencia metacognitiva asociado al uso de estrategias y tareas mientras realizaban la prueba de comprensión auditiva. 
Después de que los instrumentos fueron diseñados (prueba de comprensión auditiva y cuestionario para profesores) se procedió a su aplicación. Se dividió a la población en dos grupos de manera aleatoria y sin ningún análisis previo para la división, no se clasificó a los encuestados bajo ningún criterio con el fin de obtener datos aleatorios y de varias fuentes. Dos días seguidos para evitar la discontinuidad de la obtención de datos, fueron necesarios para aplicar los instrumentos tanto al primero como al segundo grupo respectivamente. Se aplicó en primer lugar la prueba sobre la habilidad auditiva e inmediatamente después de la prueba se aplicó el cuestionario sobre metacognición para conocer los procesos cognitivos que aplicaron antes, durante y después de esta actividad, es decir, para conocer si los estudiantes utilizan estrategias metacognitivas mientras escuchan y qué tipo de estrategias metacognitivas utilizan. Esto con el propósito de analizar si los estudiantes que utilizan las estrategias metacognitivas demuestran un alto nivel de desarrollo y por lo tanto éxito en el logro de la comprensión auditiva. Además de establecer cuáles son las estrategias metacognitivas que les permiten un alto grado de comprensión auditiva, es decir regulación y control de los procesos de escuchar una lengua extranjera.

Adicionalmente, se aplicó también el cuestionario sobre conocimiento metacognitivo a los docentes en un solo día de trabajo y posteriormente se trabajó en la tabulación y análisis de los datos obtenidos.

\section{Resultados.}

A decir de Torres (2006) “el análisis y la discusión de los resultados es el aspecto más importante que se va a tener en cuenta en toda investigación”. Pues este aspecto de la investigación debe indicar si el estudio reveló o no a las hipótesis o preguntas directrices del trabajo investigativo, e interpretar los hallazgos relacionados con el problema de la investigación. En este sentido se 
determina que después de analizar los resultados de la prueba de la habilidad auditiva destacan dos grupos el uno minoritario, con un promedio alto de respuestas correctamente contestadas y el otro que es el grupo mayoritario, con un promedio bajo de respuestas correctas. Se trabajó en correlacionar las escalas valorativas de los cinco factores del cuestionario (MALQ) con los altos promedios obtenidos por los estudiantes en la prueba auditiva, lo cual determinó que hay una diferencia significativa sobre el nivel de conciencia metacognitiva. Las escalas totalmente de acuerdo, y de acuerdo son las respuestas que este grupo con alto rendimiento muestra en el cuestionario, mientras que el grupo de bajo rendimiento en la prueba mayoritariamente responde casi en desacuerdo y en desacuerdo lo que implica que no hacen uso de las estrategias metacognitivas. El grupo de alto rendimiento en la prueba auditiva tiene la capacidad de regular su aprendizaje y demuestran ser más activos al controlar sus procesos auditivos. De este modo se concluye que el uso de las estrategias metacognitivas incide positivamente en la comprensión auditiva del inglés y que solo un grupo pequeño de la población utiliza estas estrategias al momento de realizar una tarea auditiva que bien las adquirieron durante sus experiencias o imitaciones de quienes los rodean.

A través del cuestionario (MALQ) se determinó también el tipo de estrategias metacognitivas que los estudiantes con alto rendimiento en la prueba auditiva utilizan estas son: (plan-evaluation) plan-evaluación; (directed-attention) atención dirigida; y (problem-solving) resolución de problemas. La primera estrategia se encuentra dentro de la fase de planificación; el segundo tipo de estrategia se encuentra dentro de la fase de monitoreo y el último tipo de estrategia se encuentra dentro de la fase de evaluación. Estas tres fases son el eje fundamental de para el desarrollo y aplicación de las estrategias metacognitivas que le permiten al educando desarrollar un mejor desempeño dentro de una actividad auditiva. 
En cuanto a los resultados obtenidos del cuestionario aplicado a los docentes se puede informar que luego del análisis respectivo se obtiene que los docentes conocen a gran escala lo que significa la metacognición y la aplicación de estrategias metacognitivas. La mayoría de los docentes demuestra un alto grado de conocimiento metacognitivo, sin embargo se determina un alto grupo de estudiantes que no aplican o conocen sobre estrategias metacognitivas demostrando así un bajo nivel de rendimiento en cuanto a la comprensión auditiva de la prueba. Esto indica que no hay instrucción sobre metacognición ni aplicación de estrategias metacognitivas cuando se desarrolla actividades auditivas dentro de las aulas de clase. Cabe recalcar que de acuerdo con Iguzquiza \& Rada (2001) la encuesta tiene ciertas desventajas que pueden afectar los resultados de la investigación y una de ellas es que los encuestados pueden desviar la verdad al momento de responder a las preguntas del cuestionario, por lo que bien los resultados de la prueba de comprensión auditiva y el cuestionario (MALQ) se deben a la falta de conocimiento sobre metacognición por parte de los docentes quienes no han instruido a sus estudiantes para el uso de estrategias metacognitivas que le permiten al estudiante tener un mejor desempeño en cuanto al proceso que se debe aplicar en las actividades de audición en inglés, sino que más bien se han dedicado al mero hecho de simplemente completar información o decir si determinada información es verdadera o falsa.

En conclusión, esta investigación ha servido para demostrar que la aplicación de estrategias metacognitivas de planificación, monitoreo y evaluación aportan significativamente al desarrollo de la comprensión auditiva del inglés y que propiamente las estrategias de plan-evaluación, atención dirigida y resolución de problemas les sirven mayoritariamente a los estudiantes que demostraron un alto nivel de comprensión auditiva, por lo que al ser una población homogénea y con los mismos rasgos sociales y de edad, pueden ser enseñadas también a los estudiantes con menor nivel de conocimiento y conciencia metacognitiva para enriquecer sus habilidades metacognitivas e incluso 
aplicarlas después en diferentes situaciones de aprendizaje. Por otro lado los profesores están llamados a instruir o enseñar a desarrollar estas estrategias y aplicarlas en la práctica auditiva del inglés a través de varias actividades que promueven el desarrollo metacognitivo, brindar a los estudiantes oportunidades para que aprendan a usar sus estrategias que les ayuden a controlar, regular y evaluar los procesos auditivos para ir más allá de la simple repetición de un audio.

\section{Conclusiones.}

De acuerdo con Iguzquiza \& Rada (2001) una encuesta siempre tiene cierto nivel de no autenticidad, ya que no es posible lograr total veracidad en las respuestas. Los encuestados no siempre proyectan su realidad en ella. De esto se deriva que la mayoría de los docentes encuestados demuestran que conocen sobre metacognición. Sin embargo los estudiantes no manifiestan conocimiento consciente y manejo de la metacognición, pues muy pocos logran éxito en la actividad auditiva que se les aplicó.

Por otro lado, es importante mencionar que las estrategias metacognitivas también se las adquiere de manera experiencial (Al-Alwan et al., 2013). Es decir a lo largo del aprendizaje de la vida y mediante otros individuos exitosos en los procesos de aprendizaje. Con base en lo mencionado, los pocos estudiantes que obtuvieron un buen nivel en la actividad auditiva y un nivel de acuerdo en el cuestionario metacognitivo pudieron adquirir dichos conocimientos de las experiencias propias o de otros y no necesariamente por los conocimientos que los docentes imparten sobre estrategias metacognitivas. Esta conclusión se presenta debido a que en las planificaciones y las observaciones de clase analizadas no se observa la aplicación de estas estrategias. 
Si bien los resultados de la encuesta dirigida a los profesores arrojan en general un buen nivel de conocimiento sobre metacognición, es notable en sus planificaciones que no las aplican con los estudiantes. Puede sostenerse entonces que es debido a la considerable preocupación por desarrollar las habilidades de hablar y escribir más que por el fortalecimiento de la habilidad receptiva. Vale mencionar que la habilidad auditiva precede a la productiva y de acuerdo con Sandoval (2014) primero debe desarrollarse la habilidad receptiva y luego la productiva.

Los estudiantes no logran el éxito en la prueba de comprensión auditiva, pues los resultados del cuestionario de conciencia metacognitiva sobre la mencionada prueba demuestran que no aplican acciones de planificación, control, monitoreo ni evaluación en el proceso auditivo. De esta manera se confirma que los estudiantes no están instruidos para usar dichas estrategias que mejoran sustancialmente su rendimiento en esta habilidad.

La práctica de las simples actividades cognitivas que son: conocer, analizar, inferir, memorizar o resumir determinado conocimiento no es suficiente para propender en los estudiantes un proceso de comprensión de lo que aprenden y para qué aprenden. No existe un análisis crítico y consciente que les permita responsabilizarse de su aprendizaje y la manera cómo aprenden. En otras palabras, los estudiantes no han logrado la posibilidad de conocerse a sí mismos como seres que tienen la capacidad de autorregular el proceso de desarrollo de la habilidad auditiva del inglés. Por lo tanto, se hace necesario el trabajo en actividades para el desarrollo de las destrezas metacognitivas. 


\section{Bibliografía.}

Al-Alwan, A., Asassfeh, S., \& Al-Shboul, Y. (2013). EFL Learners' Listening Comprehension and Awareness of Metacognitive Strategies: How Are They Related? International Education Studies, 6(9). http://doi.org/10.5539/ies.v6n9p31

Brown, S. (2011). Listening Myths: Applying Second Language Research to Classroom Teaching. University of Michigan Press.

Cogo, A., \& Dewey, M. (2012). Analysing English as a Lingua Franca: A Corpus-driven Investigation. Bloomsbury Publishing.

Dörnyei, Z., \& Taguchi, T. (2010). Questionnaires in Second Language Research: Construction, Administration, and Processing. Routledge.

Huseyin, O. (2005). METACOGNITION IN FOREIG/SECOND LANGUAGE LEARNING AND TEACHING. H.U. Journal of Education, 29, 147-156.

Iguzquiza, V. D. de R., \& Rada, V. D. de. (2001). Diseño y elaboración de cuestionarios para la investigación comercial. ESIC Editorial.

Jaramillo, I. D. T., \& Ramírez, R. D. P. (2006). Método y conocimiento: metodología de la investigación: investigación cualitativa/investigación cuantitativa. Universidad Eafit.

Li, W. (2013). A Study of Metacognitive Awareness of Non-English Majors in L2 Listening. Journal of Language Teaching and Research, 4(3). http://doi.org/10.4304/j1tr.4.3.504-510

Modi, D. J. (1991). Construction And Standardisation Of Listening Comprehension. Mittal Publications.

Mohamed Mahmoud Ghoneim, N. (2013). The Listening Comprehension Strategies Used by College Students to Cope with the Aural Problems in EFL Classes: An Analytical Study. English Language Teaching, 6(2). http://doi.org/10.5539/elt.v6n2p100

Núñez Perez, J. C., Solano Pizarro, P., González-Pienda, J. A., \& Rosário, P. (2006). El aprendizaje autorregulado como medio y meta de la educación. Retrieved from http://repositorium.sdum.uminho.pt/handle/1822/11868

Peña-Ayala, A. (2014). Metacognition: Fundaments, Applications, and Trends: A Profile of the Current State-Of-The-Art. Springer.

Sandoval, M. S. S. (2014). Estrategias metacognitivas en la comprensión auditiva del inglés como segunda lengua. Lenguas Modernas, (36), Pág-25.

Tamayo, M. T. y. (2004). El proceso de la investigación científica. Editorial Limusa.

Tarricone, P. (2011). The Taxonomy of Metacognition. Psychology Press. 
Vol. 3, núm. 4, octubre, 2017, pp. 426-446

Estrategias metacognitivas en la comprensión auditiva del inglés, caso colegio municipal Fernández Madrid

Torres, C. A. B. (2006). Metodología de la investigación: para administración, economía, humanidades y ciencias sociales. Pearson Educación.

Vandergrift, L., \& Goh, C. C. M. (2012). Teaching and Learning Second Language Listening: Metacognition in Action. Routledge. 